\title{
Sneddon's syndrome presenting with severe disabling bilateral headache
}

\author{
Cinzia Cavestro $\cdot$ Luca Richetta $\cdot$ Enrico Pedemonte • \\ Giovanni Asteggiano
}

Received: 7 December 2008/Accepted: 4 February 2009/Published online: 14 March 2009

(C) Springer-Verlag 2009

\begin{abstract}
Sneddon's syndrome is a rare vascular disease affecting mainly skin and brain arterioles leading to their occlusion due to excessive endothelial proliferation. The two main features of this syndrome are livedo reticularis and lacunar subcortical infarcts. Here, we describe the case of a 64-year-old woman presenting with a 4-year history of a throbbing, bilateral, parieto-occipital headache associated with facial pain, but without any other accompanying symptom. The pain, initially misdiagnosed as atypical trigeminal neuralgia, worsened up to chronic daily and such severely disabling headache that she was constrained to bed. She presented with reduced cognitive functions, diffuse and severe livedo reticularis, severe myalgias and mild stiffness. All diagnostic test for different diseases were performed and other diseases excluded except for Sneddon's syndrome. Her symptoms were reduced firstly using acetylsalicylic acid, then ticlopidine $250 \mathrm{mg}$ bid was begun and then Pentoxyphillin, resulting in a significant improvement of symptoms with the disappearance of headache. Her worsening in the first year was characterized by obsessive-compulsive behaviours, body-image misperceptions and panic attacks, improved for a period using olanzapine. Considering this case, we remark the importance of using headache classification to avoid diagnostic errors, secondly, we describe an atypical manifestation of
\end{abstract}

\section{Cavestro $(\square)$}

Department of Neurology, Headache Center,

Ospedale "San Lazzaro" A.S.L. CN2, Alba (CN), Italy

e-mail: cicaves@tin.it

\section{Richetta}

Department of Radiology, Fossano Hospital, Fossano (CN), Italy

E. Pedemonte - G. Asteggiano

Department of Neurology, "San Lazzaro" Hospital, Alba, Italy
Sneddon's syndrome and therapeutic efficacy of using ticlopidine and pentoxyphillin.

\section{Introduction}

Sneddon's syndrome is a rare, progressive, non-inflammatory vasculopathy affecting the small- and medium-size arteries of the brain and skin, leading to their occlusion due to excessive endothelial proliferation. The two main features of this syndrome are livedo reticularis and lacunar subcortical infarcts. Other frequent symptoms are dementia, heart valvulopathy, hypertension and renal failure $[1,2]$.

\section{Case report}

Here we describe the case of a 64-year-old woman presenting with a 4-year history of a de-novo throbbing, bilateral, parieto-occipital headache associated with facial pain, but without any other accompanying symptom (phono-, photophobias, nausea nor vomiting); headache worsened in orthostatism and improved in clinostatism. The pain, initially misdiagnosed as atypical trigeminal neuralgia, worsened up to chronic daily and such severely disabling headache that she was constrained to bed. Several drugs had been used in the previous 4 years, without any effect (benzodiazepine, Ca-antagonist, central smooth-relaxant, tricyclic antidepressants, antiepileptic drugs, SSRI); she treated pain daily with non-steroidal anti-inflammatory drugs or tramadol, without pain improvement.

Other symptoms were reduced cognitive functions, diffuse and severe livedo reticularis that persisted since she was a young adult (Fig. 1), and severe myalgias and mild 


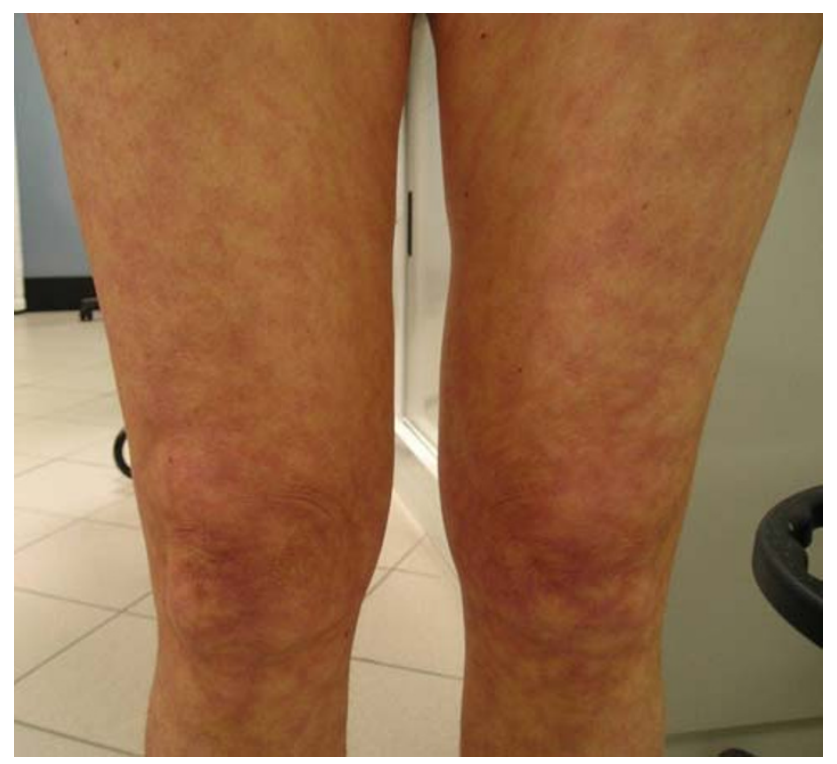

Fig. 1 Livedo reticularis in our patient

stiffness that progressively worsened in the previous years. The patient had a history of mild hypertension, well controlled by enalapril $10 \mathrm{mg}$, and a strong family history of headache. Her mother showed livedo reticularis and died when she was about 60 years old for cardiac valvulopathy and dementia. Two sisters showed mild livedo reticularis and myalgias. Physical examination revealed reduced skin elasticity with slight hypomimia, palmar erythema, malar rash, and widespread red dermographism. A pronounced livedo was present on arms, legs, trunk and neck, independently from patient position or room temperature. Neurologic examination revealed only slight lower left facial weakness and mild impairment of multiple cognitive domains (memory, attention, problem solving, and abstract reasoning). No other symptoms suggestive of particular forms of arteritis, or sleep disorders were referred. Cardiac and sovra-aortic vessels pathologies were excluded.

A general, metabolic and endocrinological blood examination was normal, antiviral antibodies (hepatitis B and $\mathrm{C}$, and HIV) were absent and serology for syphilis was negative, antiphospholipid antibodies (lupus anticoagulant, anticardiolipin antibodies, anti-beta2-glycoprotein antibodies, anti-S-protein coagulant antibodies) were absent, an immunological screening was normal (antinuclear antibodies, antineutrophil cytoplasmic antibodies, antidouble-stranded-DNA antibodies, C3, C4, cryoglobulins, serum immunoelectrophoresis) and other autoantibodies were absent; test for antiphospholipid syndrome and immunity were repeated several times, at least at 12-week intervals. A gadolinium-enhanced MRI showed the presence of wide subcortical lacunar and confluent infarcts (Fig. 2), while MR angiography sequences were unrevealing. Nerve conduction studies provided normal results.

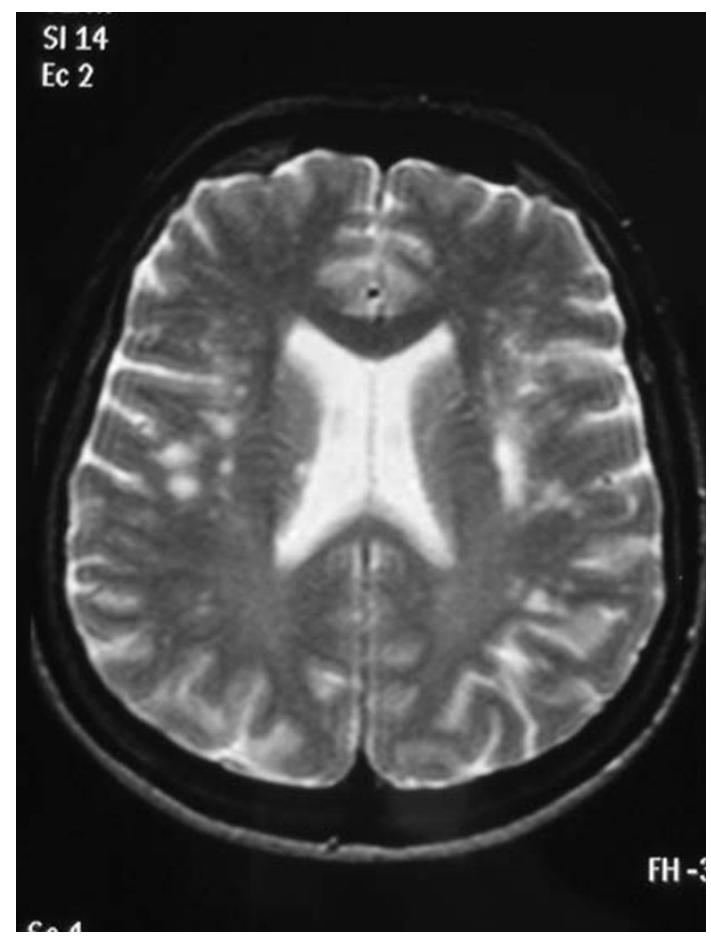

Fig. 2 MRI T2 sequence of our patient shows several lacunar lesions in the sub-cortical brainregion

Electromyogram showed only a mild and aspecific bilateral myopathic impairment from proximal arm muscles. By echocardiography, a mild mitral insufficiency was disclosed. Skin biopsy did not show any specific finding. In periumbilical fat specimens, no amyloid deposits were revealed. Genetic screening for CADASIL was negative. CSF was normal and isoelectrofocusing did not disclose any abnormal finding. We then diagnosed a Sneddon's syndrome without antiphospholipid antibodies and began treating the patient accordingly. Our patient was first treated with acetylsalicylic acid, $150 \mathrm{mg}$ daily. All the previous therapies were progressively ( 2 months) discontinued. This treatment reduced only partially symptom severity in about 1 month. Aspirin was then discontinued and ticlopidine $250 \mathrm{mg}$ bid was begun, resulting in a significant improvement of symptoms with the disappearance of headache. Pentoxyphillin was then added at the dose of $400 \mathrm{mg}$ bid. The patient then reported well-being that lasted about a month when she began to develop obsessivecompulsive behaviours, body-image misperceptions and panic attacks. These psychiatric symptoms were treated adding olanzapine, at the dose of $15 \mathrm{mg}$ daily; olanzapine dose was then gradually reduced during the following 6 months for well-being. The patient did not show headache or main cognitive impairment for the following 12 months, her livedo reticularis was less evident and only mild myalgias persist, without need for analgesics. After 12 months of well-being, quite abruptly, the patient relapsed 
showing again dementia (temporo-spatial disorientation, decreased attention, memory, logic, and apraxia), severe psychiatric symptoms, myalgias and worsened livedo reticularis, but no headache. Only a slight improvement was achieved switching antiplatelet therapy to clopidogrel $75 \mathrm{mg}$ daily, increasing pentoxiphillin up to $600 \mathrm{mg}$ tid and olanzapine up to $20 \mathrm{mg}$ daily.

\section{Discussion}

Two main observations could be inferred from this case. First, we remark the importance of using headache classification to avoid diagnostic errors. Second, we describe an atypical manifestation of Sneddon's syndrome. Headache disorders are widely described in the last international classification [3]. This classification has mainly been conceived to avoid errors and misdiagnoses. In this case the patient has not been properly treated for a 4-year period and an atypical bilateral trigeminal neuralgia was diagnosed. This is an example of how it is important to sift through a patient's signs and symptoms when diagnostic criteria are not met in order to hypothesise a different disorder and modify treatment accordingly. A few papers tried to evaluate a possible association between migraine and Sneddon's syndrome, but available literature is not conclusive [4]. Headache has never been described as the main symptom, but has only been cited as an accompanying one [2]. This case stands out for the atypical presentation with severe, persistent, very long-lasting, disabling headache, long time before other neurological symptoms and signs manifested. Mechanism of headache pathogenesis in patients with antiphospholipid antibodies or other coagulative disorders is supposed to be "vascular" but it is less clear when such antibodies are absent [4]. Classic pathologic findings are thrombosis and recanalization in medium-small vessels and meningeal vessels, both in patients with and without antiphospholipid antibodies [4]. Sneddon's syndrome is usually treated with antiplatelet or anticoagulant therapy, depending mainly on the presence or absence of antiphospholipid antibodies. A recent review does not show any significant difference in the prognosis of the patients following the two treatments [1]. In our case antiplatelet therapy was crucial and was associated with a durable painfree period. Unfortunately, Sneddon's syndrome is a progressive disease and no causative therapy is yet known.

Conflict of interest None.

\section{References}

1. Francès C, Papo T, Wechsler B, Laporte J, Biousse V, Piette J (1999) Sneddon syndrome with or without antiphospholipid antibodies: a comparative study in 46 patients. Medicine 78:209_ 219

2. Boesch SM, Plörer AL, Auer AJ, Poewe W, Aichner FT, Felber SR, Sepp NT (2003) The natural course of Sneddon syndrome: clinical and magnetic resonance imaging findings in a prospective six year observation study. J Neurol Neurosurg Psychiatry 74: 542-544

3. Headache Classification Subcommittee of The International Headache Society (2004) The International Classification of Headache Disorders, 2nd edn. Cephalalgia 24 (suppl 1):1-160

4. Tietjen GE, Al-Qasmi MM, Gunda P, Herial NA (2006) Sneddon's syndrome: another migraine-stroke association? Cephalalgia $26: 225-232$ 